\title{
Phytochemical Composition, Biological Activities of Croton lobatus L. Leaves, Hydrolysis Effect on Activities and Chemical Composition
}

\author{
Marthe Dominique Chodaton-Zinsou*, Fidèle Mahoudo Assogba, Eléonore Yayi-Ladékan, \\ Fernand Gbaguidi, Mansourou Moudachirou, Joachim Djimon Gbénou
}

Faculty of Sciences and Technics, University of Abomey-Calavi, Abomey-Calavi, Benin

Email address:

marthezinsou@hotmail.fr (M. D. Chodaton-Zinsou)

${ }^{*}$ Corresponding author

To cite this article:

Marthe Dominique Chodaton-Zinsou, Fidèle Mahoudo Assogba, Eléonore Yayi-Ladékan, Fernand Gbaguidi, Mansourou Moudachirou, Joachim Djimon Gbénou. Phytochemical Composition, Biological Activities of Croton lobatus L. Leaves, Hydrolysis Effect on Activities and Chemical Composition. American Journal of Applied Chemistry. Vol. 8, No. 1, 2020, pp. 13-22. doi: 10.11648/j.ajac.20200801.13

Received: February 5, 2020; Accepted: February 27, 2020; Published: March 10, 2020

\begin{abstract}
Croton lobatus $L$. is medicinal plant widely used in traditional medicine, but little known in literature for its biological properties. Aims were to study its phytochemical composition and some biological properties and influence of acid hydrolysis on biological activities and chemical composition of Croton lobatus $L$. extracts. Successive extractions with solvents of increasing polarity (cyclohexane, ethyl acetate, and methanol) were carried out on powder of Croton lobatus L. leaves. Evaluation of xanthine oxidase, acetylcholinesterase, 5-lipoxygenase and $\alpha$-amylase inhibitions, antioxidant activity was performed. Acid hydrolysis effect was evaluated by comparison of chemical composition and xanthine oxidase, $\alpha$-amylase inhibition activities of hydrolyzed and unhydrolyzed extracts. Results showed that at $50 \mathrm{mg} / \mathrm{L}$ Croton lobatus $L$. has very small antioxidant activity (3.4 $4.9 \%)$, small inhibitory activity of 5-lipoxygenase (6.3-6.9\%), inhibitory activity of acetylcholinesterase between (2.7 - 31.3\%), moderate inhibitory activity of $\alpha$-amylase $(52.8-64.3 \%)$, moderate inhibitory activity of xanthine oxidase (22.2 - 62.6\%). Croton lobatus $L$. could be used in diabetes and gout treatments, given results of $\alpha$-amylase inhibition and xanthine oxidase. Acid hydrolysis has negative effect on process of xanthine oxidase inhibition and on chemical composition.
\end{abstract}

Keywords: Croton lobatus L., Antioxidant, Acetylcholinesterase, Xanthine Oxidase, $\alpha$-amylase, 5 -Lipoxygenase, Inhibition, Acid Hydrolysis

\section{Introduction}

Croton lobatus L. called "alovi Aton" by people of Benin southern was an annual herbaceous plant $30-60 \mathrm{~cm}$ tall belonging to Euphorbiaceae family. Iwast met from Senegal to Cameroon, in Egypt, Ethiopia, Sudan and Arabia [1]. It was widely used in traditional medicine in treating spasms, threats, abortion and hypertension [2]. Croton lobatus L. is one of plants few studied for their biological properties. Previous studies on plant led to isolation of alkaloids [3] and evaluation of antimicrobial activity [4-6]. The objective of this study was to evaluate its phytochemical composition, antioxidant activity, inhibitory activities of $\alpha$-amylase, xanthine oxidase, 5-lipoxygenase, acetylcholinesterase and then to study influence of acid hydrolysis on some properties and on chemical composition.

\section{Materials and Methods}

\subsection{Plant Material}

Plant material consisted in Croton lobatus L. leaves harvested at Abomey-Calavi in the southwest of Republic of Benin. Plant was identified at National Herbarium of University of Abomey-Calavi under number AA 6542/HNB where voucher specimen was conserved.

\subsection{Chemicals and Reagents}

Chemicals and solvents used were analytical grade and purchased from Sigma-Aldrich-Fluka (Saint-Quentin, France). 


\subsection{Extracts Preparation}

Cyclohexane, dichloromethane, ethyl acetate and methanol extracts were prepared using method cited in ChodatonZinsou [7].

\subsection{Chemical Composition}

Chemical composition (phenolic compounds, flavonoids, tannins, anthocyanins) was determined following methods cited in Chodaton-Zinsou [7].

\subsection{Biological Activities}

Antioxidant, anti-inflammatory activities, acetylcholinesterase, xanthine oxidase, $\alpha$-amylase inhibition activities essays were cited in Chodaton-Zinsou [7].

\subsection{Extract Hydrolysis}

Extract hydrolysis was performed according to method developed by Wach [8]. $20 \mathrm{mg}$ of each extract were dissolved in $1 \mathrm{~mL}$ of suitable solvent and heated for 30 minutes at $100^{\circ} \mathrm{C} .200 \mu \mathrm{L}$ of that solution were collected and evaporated to dryness. Then extract obtained was dissolved in $100 \mu \mathrm{L}$ of $3 \mathrm{M} \mathrm{HCl}-$ methanol mixture $(60: 40, \mathrm{v} / \mathrm{v})$, heated at $100^{\circ} \mathrm{C}$ for 20 minutes and evaporated to dryness.

\subsection{Derivatization and GC-MS Analysis}

Derivatization and GC analysis were carried out following methods cited in Chodaton-Zinsou [7].

\section{Results and Discussion}

\subsection{Phenolic Compounds, Flavonoids, Tannins, and Anthocyanins Contents}

Results of quantification of phenolic compounds, flavonoids, tannins and anthocyanins (table 1) showed that these extracts contain few phenolic compounds. Methanolic extract contains more polyphenols than the others and cyclohexane extract contains less. These levels would influence biological activities, in particular antioxidant activity.

Table 1. Phytochemical composition of Croton lobatus L. leaves

\begin{tabular}{lllll}
\hline Extracts (50 mg/L) & Polyphenols (mg eq AG/g) & Flavonoids (mg EQ/g) & Tannins (mg ECat/g) & Anthocyanins (mg Eq C3G/kg) \\
\hline Cyclohexane & $2.3 \pm 0.1$ & $\mathrm{Nd}$ & $\mathrm{Nd}$ & $0.0015 \pm 0$ \\
Dichloromethane & $15.8 \pm 0.6$ & $\mathrm{Nd}$ & $\mathrm{Nd}$ & $0.0025 \pm 0$ \\
Ethyl acetate & $17.3 \pm 0.3$ & $6.3 \pm 0.2$ & $\mathrm{Nd}$ & $0.0005 \pm 0$ \\
Methanol & $20.2 \pm 0.7$ & $0.8 \pm 0.1$ & $\mathrm{Nd}$ & $\mathrm{Nd}$ \\
\hline
\end{tabular}

Nd: no determined

\subsection{Antioxidant Activity}

Results obtained (table 2) show that plant has a very weak antioxidant activity compared to ascorbic acid activity which is reference used. Antioxidant activity of leaves varies from 3.4 to $4.9 \%$ while ascorbic acid at $5.3 \mathrm{mg} / \mathrm{L}$ was active at $53.01 \%$. Plant therefore contains very few antioxidants responsible for activity. This would be confirmed by low content of polyphenols (table 1) which are antioxidants. This weak antioxidant activity seems to be specific to species of genus Croton. Thus, antioxidant activity of Croton caudatum was $83.29 \%$ with a concentration of $40 \mathrm{mg} / \mathrm{mL}(40000 \mathrm{mg} / \mathrm{L})$ [9]. That of Croton bonplandianum, at $60 \mu \mathrm{g} / \mathrm{mL}$ was $19.06 \pm$ $0.12 \%$ [10]. Another Croton bonplandianum species, at 0.5 $\mathrm{mg} / \mathrm{mL}(5000 \mathrm{mg} / \mathrm{L})$ exhibited $59.62 \%$ activity [11].

\subsection{Lipoxygenase Inhibition}

Croton lobatus $L$. showed a very small 5-lipoxygenase inhibitory activity compared to reference which was NDGA (table 2). This activity of Croton lobatus $L$. varies between $6.3 \%$ and $6.9 \%$. To our knowledge, there is no investigation in literature on its ability to inhibit 5-lipoxygenase activity. On other hand, in vivo studies have been carried out with Croton argyrophyllus [12] and Croton leptostachyus Kunth [13]. These studies focused on edema inhibition on laboratory's rat and showed its anti-inflammatory activity. Our Croton lobatus L. was therefore not a potential inhibitor of 5-lipoxygenase.

\subsection{Acetylcholinesterase Inhibition}

Table 2 which presented, among others things, acetylcholinesterase inhibition results showed that apolar extracts had a very small activity. The most polar extracts, on the other hand, had moderate activity. Other Croton species were more active than Croton lobatus L. Thus, leaves of Croton zambesicus $(42.5 \mu \mathrm{g} / \mathrm{mL})$ have $51.29 \pm 3.86 \%$ of inhibition [14]. Extraction solvents do not influence activity within Croton genus. Indeed, activity of dichloromethane extract of Croton lobatus L. $(2.7 \pm 0.3 \%)$ was much lower than that of dichloromethane extract of Croton socotramus bark $(0.05 \mathrm{mg} / \mathrm{ml})$ which is $40.61 \%$ and methanolic extract at the same concentration has zero activity on this enzyme [15]. Edapho-climatic conditions, extraction technic could explain these differences. To our knowledge, this study on acetylcholinesterase inhibition was the first for Croton lobatus $L$.

\section{5. o-amylase Inhibition}

Evaluation of $\alpha$-amylase inhibition activity of Croton lobatus $L$. extracts was performed. Results obtained (table 2) showed that all extracts $(50 \mathrm{mg} / \mathrm{L})$ exhibit $\alpha$-amylase inhibition activity with percentages greater than $50 \%$. The lower percentage was obtained with cyclohexane extract $(52.8 \pm 0.4 \%)$ followed by methanol extract $(61.4 \pm 1.0 \%)$, 
dichloromethane extract $(63.1 \pm 2.5 \%)$ and ethyl acetate extract $(64.3 \pm 2.4 \%)$. Acarbose $(50 \mathrm{mg} / \mathrm{L})$ which was standard drug of $\alpha$ - amylase inhibition used presented 56,4 \pm $2,6 \%$ of inhibition. The two most polar extracts were more effective than positive control used: acarbose. Results showed that Croton lobatus L. contains $\alpha$-amylase inhibitors. These results could be attributed to an abundance of inhibitors such as, saponins, steroids, alkaloids, terpenoids $[16,17]$ responsible of this property.

Note that no data on Croton lobatus L. property to inhibit $\alpha$-amylase in literature that can allow comparison exists. To our knowledge, results of our work represent the first report.

\subsection{Xanthine Oxidase Inhibition}

Croton lobatus $L$. extracts were tested for their activities on xanthine oxidase. Results of that evaluation (table 2) showed that only two more polar extracts $(50 \mathrm{mg} / \mathrm{L})$ had higher inhibition percentages than $50 \%$. The highest inhibition was achieved with methanol extract $(62.6 \pm 1.8 \%)$ while the lowest inhibition was obtained with cyclohexane extract $(22.2 \pm 1.1 \%)$. Ethyl acetate extract presented $50.5 \pm 0.9 \%$ of inhibition. Percentages obtained with ethyl acetate and methanol extracts could be explained by abundance or xanthine oxidase inhibitors quality such as polyphenols, flavonoids or saponins which are xanthine oxidase inhibitors $[17,18]$.

Table 2. Croton lobatus L. biological activities.

\begin{tabular}{|c|c|c|c|c|c|}
\hline \multirow[b]{2}{*}{ Extracts $(50 \mathrm{mg} / \mathrm{L})$} & \multicolumn{5}{|c|}{ Activities (\%) } \\
\hline & Antioxidant & $\begin{array}{l}\text { acetylcholinesterase } \\
\text { inhibition }\end{array}$ & $\begin{array}{l}\text { 5-lipoxygenase } \\
\text { inhibition } \\
\end{array}$ & $\begin{array}{l}\text { Xanthine oxidase } \\
\text { inhibition }\end{array}$ & $\begin{array}{l}\alpha \text {-Amylase } \\
\text { inhibition }\end{array}$ \\
\hline Cyclohexane & $3.4 \pm 0.2$ & $6.0 \pm 0.3$ & $6.7 \pm 0.3$ & $22.2 \pm 1.1$ & $52.8 \pm 0.4$ \\
\hline Dichloromethane & $4.2 \pm 0.2$ & $2.7 \pm 0.3$ & $6.3 \pm 0.2$ & $19.3 \pm 0.8$ & $63.1 \pm 2.5$ \\
\hline Ethyl acetate & $4.4 \pm 0.3$ & $31.2 \pm 1.5$ & $6.9 \pm 0.3$ & $50.5 \pm 0.9$ & $64.3 \pm 2.4$ \\
\hline Methanol & $4.9 \pm 0.1$ & $31.3 \pm 1.0$ & $6.6 \pm 0.2$ & $62.6 \pm 1.8$ & $61.4 \pm 1.0$ \\
\hline Ascorbic acid $(5,3 \mathrm{mg} / \mathrm{L})$ & $53.01 \pm 0.4$ & - & - & - & - \\
\hline NDGA (4 mg/L) & - & - & $81.9 \pm 0.2$ & - & - \\
\hline Allopurinol (1 mg/L) & - & - & - & $52 \pm 1.0$ & - \\
\hline Acarbose $(50 \mathrm{mg} / \mathrm{L})$ & - & - & - & - & $56.4 \pm 2.6$ \\
\hline
\end{tabular}

NDGA $=$ Nordihydroguaiaretic acid

\section{7. o-amylase Inhibition by Hydrolysed Extracts}

Cyclohexane, ethyl acetate, methanol hydrolysed extracts were tested for their properties to inhibit $\alpha$-amylase. Results were presented on table 3 . Results showed that all hydrolysed extracts showed activity. Methanol extract was most active with $73.8 \pm 2.5 \%$ inhibition and cyclohexane extract was least active $35 \pm 4.5 \%$. A comparison of extracts activity with acarbose activity showed that ethyl acetate extract and methanol extracts inhibited more than acarbose which is positive control used. Hydrolysed extracts like unhydrolysed extracts contain $\alpha$-amylase inhibitors.

Table 3. Results of $\alpha$ - amylase inhibition by hydrolysed and non-hydrolysed extracts of Croton lobatus L. leaves.

\begin{tabular}{lll}
\hline \multirow{2}{*}{ Extracts (50 $\mathbf{~ m g} / \mathbf{L})$} & \multicolumn{2}{c}{$\boldsymbol{\alpha}$ - amylase inhibition percentage } \\
\cline { 2 - 3 } & Before hydrolysis & After hydrolysis \\
\hline Cyclohexane & $52.8 \pm 0.4$ & $35 \pm 4.5$ \\
Ethyl acetate & $64.3 \pm 2.4$ & $68.9 \pm 1$ \\
Methanol & $61.4 \pm 1.0$ & $73.8 \pm 2.5$ \\
Acarbose $(50 \mathrm{mg} / \mathrm{L})$ & $56,4 \pm 2,6$ & - \\
\hline
\end{tabular}

\subsection{Xanthine Oxidase Inhibition by Hydrolysed Extracts}

Croton lobatus L. hydrolysed extracts were tested for their ability to inhibit xanthine oxidase. Table 4 showed results. Methanol extract showed the best inhibition (31.02 $\pm 0.4 \%)$. Cyclohexane extract presented the weakest inhibition activity with $9.8 \pm 0.9 \%$. These results showed that hydrolysed extracts like unhydrolysed extracts have inhibitory properties on xanthine oxidase. This property could be explained by phenolics which, about literature [19] were inhibitors of this enzyme.

Table 4. Results of xanthine oxidase inhibition by hydrolysed and nonhydrolysed extracts of Croton lobatus L. leaves.

\begin{tabular}{lll}
\hline Extracts $(\mathbf{5 0} \boldsymbol{\mu g} / \mathbf{m L})$ & \multicolumn{2}{l}{ Xanthine oxidase inhibition percentage } \\
\cline { 2 - 3 } and reference & Before hydrolysis & After hydrolysis \\
\hline Cyclohexane & $22.2 \pm 1.1$ & $23.3 \pm 0.9$ \\
Ethyl acetate & $50.5 \pm 0.9$ & $46.2 \pm 3.7$ \\
Methanol & $62.6 \pm 1.8$ & $54.62 \pm 0.4$ \\
Allopurinol $(1,2$ & $52 \pm 1,0$ & - \\
$\mu \mathrm{g} / \mathrm{mL})$ & & \\
\hline
\end{tabular}

\subsection{Acid Hydrolysis Effect on $\alpha$-amylase Inhibition and on Xanthine Oxidase Inhibition}

A Comparison of results of table 3 showed a slight increase of inhibitory activity after hydrolysis of ethyl acetate and methanol extracts, and a decrease of inhibitory activity of cyclohexane extract. This increase can be attributed to compounds action released after hydrolysis, so an increase inhibitors $\alpha$-amylase. Decrease in inhibitory activity of cyclohexane extract may be the result of inactivity of novel compounds obtained from hydrolysis. Hydrolysis has positive effect on $\alpha$-amylase inhibition.

Results of table 4 revealed that ethyl acetate and methanol hydrolysed extracts contain less inhibitors while cyclohexane extract contains a little more. Acid hydrolysis has resulted in the decrease of hydrolysed extracts activities. This decrease in activity could be attributed to disappearance of some compounds responsible for activity present in extract before hydrolysis and also to inactivity of novel compounds derived 
from hydrolysis.

\subsection{Acid Hydrolysis Effect on Chemical Composition of Extracts}

Unhydrolysed and hydrolysed extracts of Croton lobatus L. are analyzed by GC-MS before and after derivatization to evaluate hydrolysis effect. Compounds identified in both cases were in tables 5 and 6 . Tables showed that before derivatization, 20 compounds (table 5) were identified in unhydrolysed extracts and no compound (table 6) was identified in hydrolysed extracts. After derivatization, 32 compounds (table 5) were identified in unhydrolysed extracts and 19 compounds (table 6) in hydrolysed extracts. Comparison of results before and after acid hydrolysis showed that hydrolysis process has an effect on extracts composition. Hydrolysis causes degradation of many bioactive compounds. The proof is that no compound has been identified in hydrolyzed extracts before derivatisation. Temperature $\left(100^{\circ} \mathrm{C}\right)$ heating could be responsible of this degradation.

Table 5. Compounds identified in Croton lobatus L. extracts.

\begin{tabular}{|c|c|c|c|c|c|c|c|}
\hline $\mathbf{N}^{\circ}$ & $\operatorname{Tr}(\min )$ & Compounds identified & Structure & $\begin{array}{l}\text { Cyclo hexane } \\
\text { extract }\end{array}$ & $\begin{array}{l}\text { Dichloromethane } \\
\text { extract }\end{array}$ & $\begin{array}{l}\text { Ethyl acetate } \\
\text { extract }\end{array}$ & $\begin{array}{l}\text { Methanol } \\
\text { extract }\end{array}$ \\
\hline \multicolumn{8}{|c|}{ Before derivatization } \\
\hline 1 & 6.13 & 2-Methyl Cyclopentanol & & + & & & \\
\hline 2 & 10.73 & 2-Dodecene (E)- & & & + & & \\
\hline 3 & 12.73 & 5-Tetradecen & & & & + & \\
\hline 4 & 12.75 & Undecanol & & & + & & \\
\hline 5 & 13.96 & $\begin{array}{lcc}2, \quad 6-b i s \quad(1, & 1- \\
\text { dimethylethyl)-Phenol }\end{array}$ & & & + & & \\
\hline 6 & 13.72 & (-)-Zingiberene & & +++ & & & \\
\hline 7 & 13.85 & Cis $\alpha$-bizabolene & & + & & & \\
\hline 8 & 13.98 & $\beta$-Sesquiphellandrene & & + & & & \\
\hline 9 & 14.45 & 7-Hexadecene (Z)- & & & ++ & + & \\
\hline 10 & 15.96 & 1-Hexadecanol & & & ++ & + & \\
\hline 11 & 16.02 & Phytol acetate & & & & & ++++ \\
\hline 12 & 16.25 & $\begin{array}{l}\text { 3, 7, 11, 15-Tetramethyl- } \\
\text { 2-hexadecen-1-ol }\end{array}$ & & ++++ & ++++ & ++++ & + \\
\hline 13 & 16.43 & $\begin{array}{l}\text { 3, 7, 11, 15-tetramethyl- } \\
\text { 2-hexadecen-1-ol } \\
\text { isomere } 2\end{array}$ & & + & + & & + \\
\hline
\end{tabular}




\begin{tabular}{|c|c|c|c|c|c|c|c|}
\hline $\mathbf{N}^{\circ}$ & $\operatorname{Tr}(\min )$ & Compounds identified & Structure & $\begin{array}{l}\text { Cyclo hexane } \\
\text { extract }\end{array}$ & $\begin{array}{l}\text { Dichloromethane } \\
\text { extract }\end{array}$ & $\begin{array}{l}\text { Ethyl acetate } \\
\text { extract }\end{array}$ & $\begin{array}{l}\text { Methanol } \\
\text { extract }\end{array}$ \\
\hline \multicolumn{8}{|c|}{ Before derivatization } \\
\hline 14 & 16.56 & $\begin{array}{l}\text { 3, 7-Dimethyldodeca-6- } \\
\text { 11-dien-1-ol }\end{array}$ & & + & & & \\
\hline 15 & 17.33 & 10-Heneicosene $(\mathrm{c}, \mathrm{t})$ & & & ++ & + & \\
\hline 16 & 18.15 & Phytol & & & + & + & \\
\hline 17 & 19.82 & 1-Decanol 2-hexyl & & & + & & \\
\hline 18 & 19.91 & $\begin{array}{l}\text { Hexanedioic acid, } \\
\text { dioctyl ester }\end{array}$ & & & + & & \\
\hline 19 & 21.24 & $\begin{array}{l}\text { Phthalic acid di } \\
\text { propylpentyl) ester }\end{array}$ & & & ++ & & \\
\hline 20 & 24.40 & Trans-farnesol & & + & & & \\
\hline \multicolumn{8}{|c|}{ After derivatization } \\
\hline 21 & 10.72 & Norvaline & & & & & + \\
\hline $\mathbf{N}^{\circ}$ & $\operatorname{Tr}(\min )$ & Compounds identified & Structure & $\begin{array}{l}\text { Cyclo hexane } \\
\text { extract }\end{array}$ & $\begin{array}{l}\text { Dichloromethane } \\
\text { extract }\end{array}$ & $\begin{array}{l}\text { Ethyl acetate } \\
\text { extract }\end{array}$ & $\begin{array}{l}\text { Methanol } \\
\text { extract }\end{array}$ \\
\hline \multicolumn{8}{|c|}{ After derivatization } \\
\hline 22 & 11.33 & Glycerol & & + & + & ++++ & + \\
\hline 23 & 11.53 & 1, 2, 3-Butanetriol & & & & + & \\
\hline 24 & 11.61 & Proline & & & & &..++ \\
\hline 25 & 11.64 & 2-mono-isobutyrin & & & & + & \\
\hline 26 & 11.76 & $\begin{array}{l}3, \quad 6, \quad 9, \quad 12, \quad 15- \\
\text { pentaoxaheptadecanedioi } \\
\text { c acid }\end{array}$ & & & & + & \\
\hline 27 & 12.56 & 6-Azauracyl & & & & + & \\
\hline 28 & 13.34 & Malonic acid & & + & + & & + \\
\hline
\end{tabular}




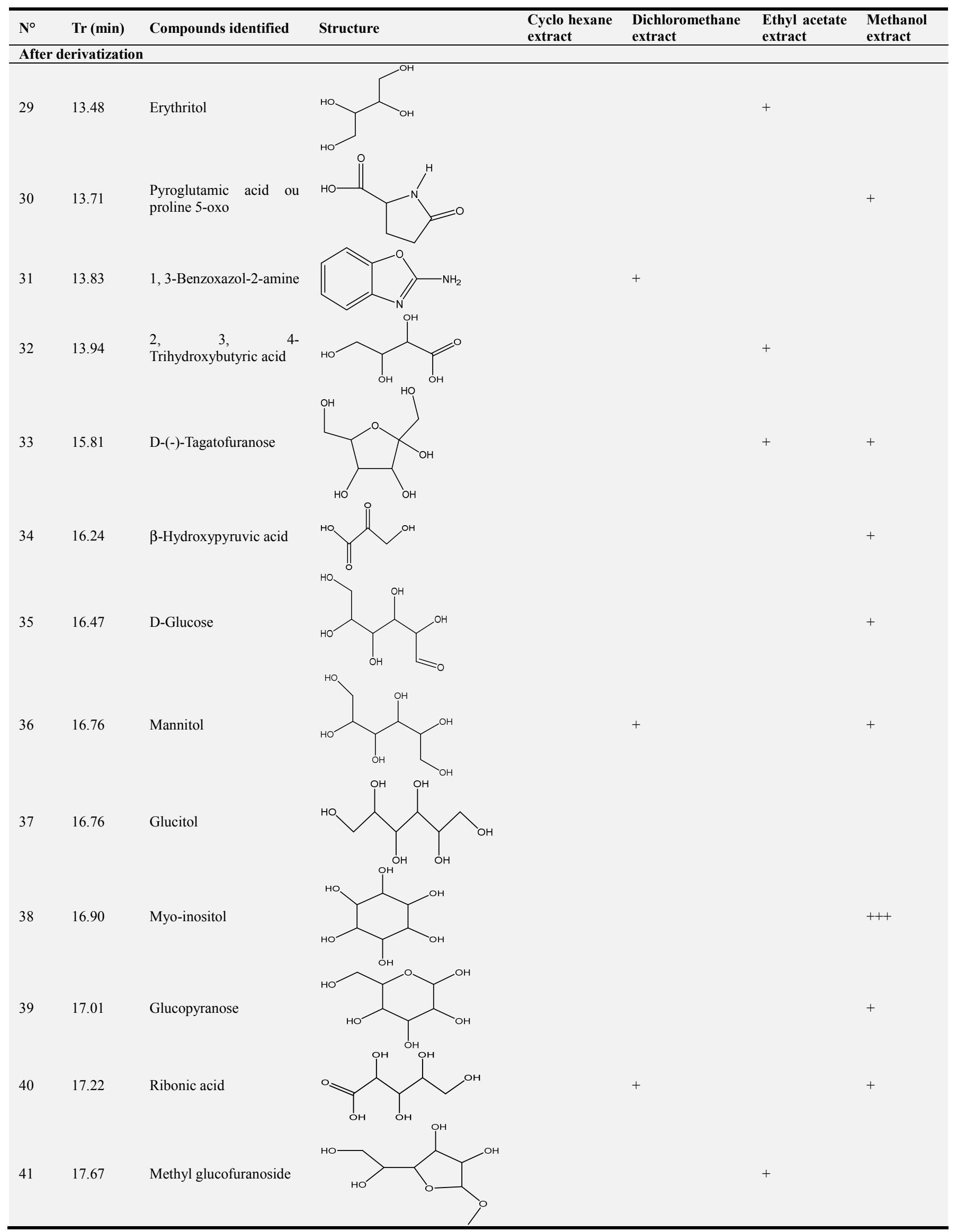




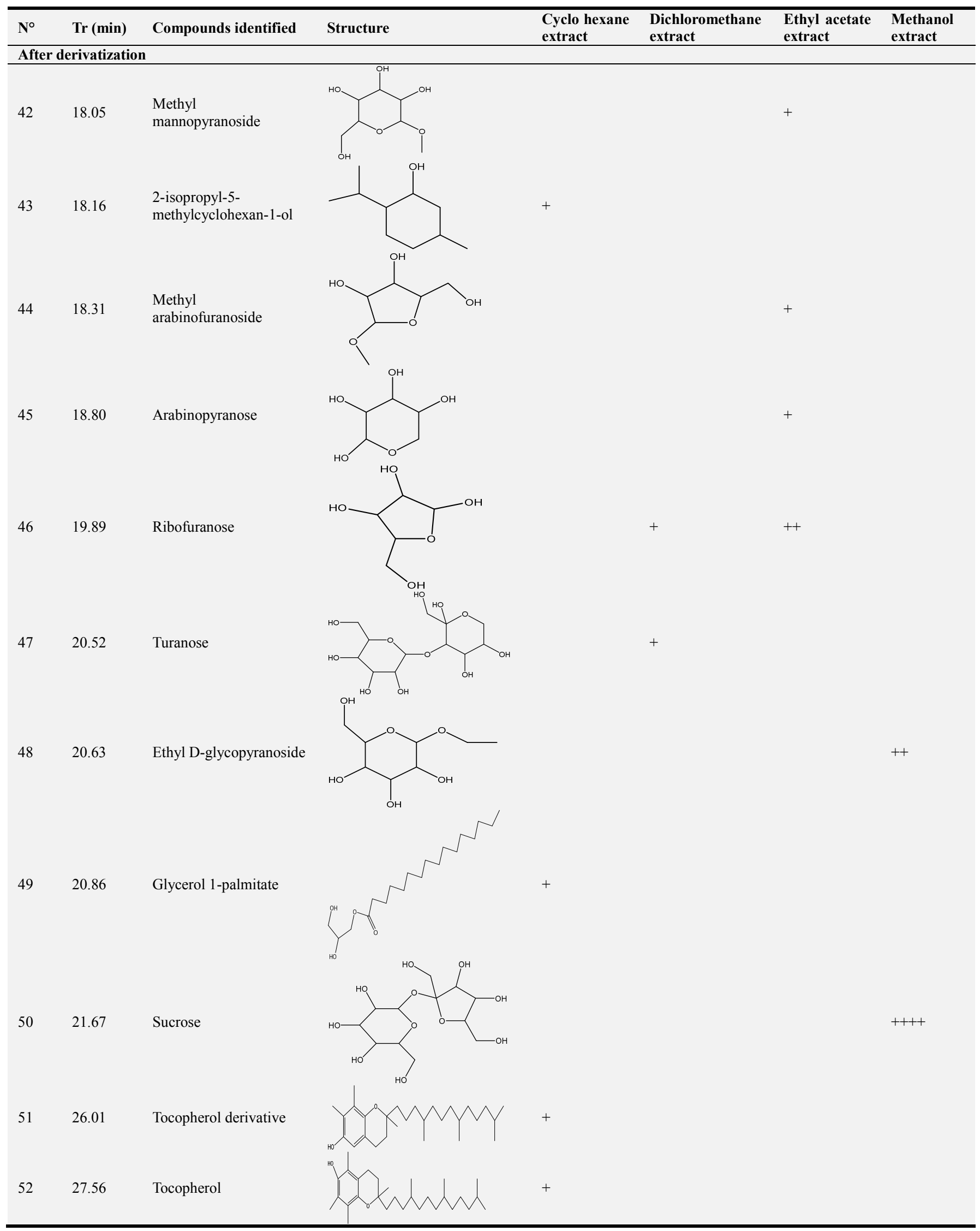

$+=$ very small presence $++=$ small presence $+++=$ presence $++++=$ strong presence 
Table 6. Compounds identified in Croton lobatus L. hydrolysed extracts.

\begin{tabular}{|c|c|c|c|c|c|c|c|}
\hline $\mathbf{N}^{\circ}$ & $\operatorname{Tr}(\min )$ & Compounds identified & Structure & $\begin{array}{l}\text { Cyclo hexane } \\
\text { extract }\end{array}$ & $\begin{array}{l}\text { Dichlorometha } \\
\text { ne extract }\end{array}$ & $\begin{array}{l}\text { Ethyl acetate } \\
\text { extract }\end{array}$ & $\begin{array}{l}\text { Methanol } \\
\text { extract }\end{array}$ \\
\hline \multicolumn{8}{|c|}{ After derivatization } \\
\hline \multicolumn{8}{|c|}{ Before derivatization } \\
\hline & & Nothingness & & & & & \\
\hline 1 & 11.33 & Glycerol & & + & ++++ & ++ & + \\
\hline 2 & 13.31 & Malic acid & & & & & + \\
\hline 3 & 13.48 & Meso-erythritol & & & & + & \\
\hline 4 & 15.78 & 1, 5-Anhydro-sorbitol & & & & + & \\
\hline 5 & 15.89 & D-(+)-Talofuranose & & & + & & + \\
\hline 6 & 15.95 & D-(-)-Fructofuranose & & & & & + \\
\hline 7 & 16.12 & $\alpha$-DL-Lyxopyranose & & & + & + & \\
\hline 8 & 16.21 & $\begin{array}{l}\text { 17-(1, 2-dihydroxyethyl)- } \\
\text { 10, 13- } \\
\text { dimethylhexadecahydro- } \\
\text { 1H-cyclopenta [a] } \\
\text { phenanthrene-3, 17-diol }\end{array}$ & & & & & + \\
\hline 9 & 16.44 & Mannopyranose & & & & & ++++ \\
\hline 10 & 16.50 & Allofuranose & & & ++ & + & \\
\hline 11 & 16.55 & $\begin{array}{l}\text { Methyl } \alpha \text {-D- } \\
\text { mannopyranoside }\end{array}$ & & & + & + & \\
\hline 12 & 16.73 & Mannitol & & & & & + \\
\hline
\end{tabular}




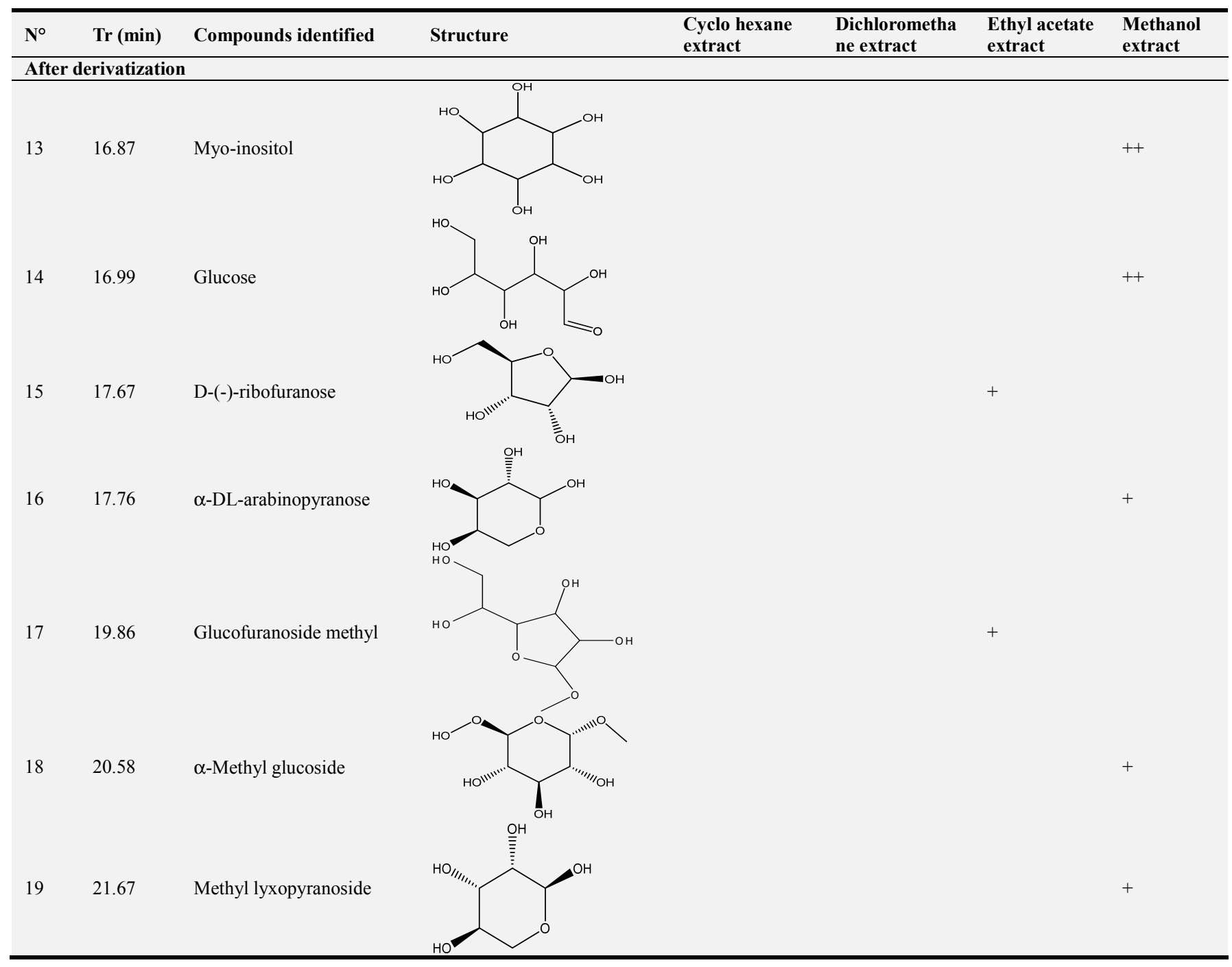

$+=$ very small presence $++=$ small presence $+++=$ presence $++++=$ strong presence

All compounds were not present in all samples: all solvents do not extract the same compounds. Some compounds were extracted by a single solvent which explains their presence in one extract. Others were present in several extracts. Presence of the same compound in several previews may be explained by the fact that these compounds could not be completely removed by the lower polarity solvent. Some compounds were present in extracts unhydrolysed and hydrolysed extracts, indicating that these compounds do not undergo hydrolysis process. This is case of D-glucose, mannitol, myo-inositol, methyl glucofuranoside, methyl mannopyranoside, arabinopyranose, ribofuranose.

\section{Conclusion}

This study on in vitro evaluation of antioxidant activity, inhibitory activities on acetylcholinesterase, xanthine oxidase, $\alpha$-amylase, 5 -lipoxygenase of Croton lobatus $L$, and on evaluation of acid hydrolysis effect on biological activities and on chemical composition is the first. The results show that Croton lobatus L. has very small antioxidant activity, small inhibitory activity of 5-lipoxygenase, moderate inhibitory activity of acetylcholinesterase. Plant could be very effective in diabetes, gout treatment. Acid hydrolysis sometimes has a negative effect on biological properties and on chemical composition.

\section{Conflict of Interest}

We declare that there is no conflict of interest

\section{Acknowledgements}

We thank the Government of Benin for awarding the scholarship that made this study possible.

\section{References}

[1] Akoègninou A., Van der Burg W. J., Van der Maesen L. J. G. Backhuys Flore analytique du Bénin, 2006, 1034 pages. 
[2] Adjanohoun E. J, Adjakidje V., Ahyi, M. R. A., Aké assi, L., Akoegninou, A., D'Almeida, J., Apovo, F., Boukef, K., Chadare, M., Cusset, G., Dramane, K., Eyme, J., Gassita, J.N., Gbaguidi, N., Goudote, E., Guinko, P., Houngnon, P., Lo, I., Keita, A., Kiniffo, H., Kone-Bamba, D., Musampa Nseyya, A., Saadou, M., Sodogandji, T., De Souza, S., Tchabi, A., Zinsou Dossa, C. \& Zohoun, T. Contribution aux études ethnobotaniques et floristiques en République Populaire du Bénin. Médecine traditionnelle et pharmacopée, Agence de Coopération Culturelle et Technique, 1989, Paris.

[3] Attioua B. K., Harisolo R., Boti J. B., Adiko V. A., Tonzibo F. Z., Djakoure L. A. Isolation and identification of alkaloids from Croton lobatus, Int. J. Pharm. Sci. Rev. Res., 2012, 13 (2), 1-4.

[4] Weniger B., Lagnika L., Ndjakou B. L., Vonthron C., L'ethnopharmacologie et la recherche de molécules antipaludéennes dans la biodiversité ivoirienne, béninoise et camerounaise. Ethnopharmacologia 2008, 41, 62-70.

[5] Lagnika L., Weniger B., Vonthron-Senecheaub C., Sanni A.; Antiprotozoal activities of compounds isolated from Croton lobatus L. Afr. J. Infect. Dis. 2009, 3 (1): 1 - 5.

[6] Lagnika L., Anago E. and Sanni A., Screening for antibacterial, antioxidant activity and toxicity of some medicinal plants used in Benin folkloric medicine, Journal of Medicinal Plants Research 2011, 5 (5), 773-777.

[7] Chodaton-Zinsou M. D. Assogba F. M., Gbaguidi F., Moudachirou M., Chemical composition and in vitro investigation of biological activities of Hemizygia bracteosa (Benth) Briq leaves, J. Pharmacognosy Phytother, 2018, 11-20.

[8] Wach A., Pyrzynska K., Biesaga M., Quercetin content in some food and herbal samples, Food chemistry, 2007 (100) 699-704.

[9] Deore S. L., S. S. Khadabadi B. A., Baviskar S. S., Khadabadi R. A., Khangenbam U. S., Koli N. P., Daga P. A., Gadbail P. A. Jain In vitro Antioxidant activity and Phenolic Content of Croton caudatum, Inter. J. Chem Tech Research, 2009, 1 (2), 174-176.

[10] Satya K., Srinivas K., Srilakshmi B. J., Lakshmi C. M., Krishna S. A, Phytochemical and pharmacoligical evaluation of Euphorbiaceae family plant leaves Acalypha indica, Croton bonplandianum Baill, Mintage J. Pharm. Med. Sci., 2015, 4 (3), $17-22$.
[11] Muhammad Naeem Qaisar, Bashir Ahmad Chaudary, Muhammad Uzair, Sajid Nawaz Hussain Evaluation of Antioxidant and Cytotoxic Capacity of Croton bonplandianum. Baill, Am. J. Plant Sci., 2013, 4, 1709-1712.

[12] José-Mirabeau O. Ramos, Cliomar A. Santos, Danielle G. Santana, Darlisson A. Santos, Péricles B. Alves, Sara M. Thomazzi, Chemical constituents and potential anti inflammatory activity of the essential oil from the leaves of Croton argyrophyllus, Rev. Bras. Farmacogn. Braz. J. Pharmacogn. 2013, 23 (4), 644-650.

[13] Wilmer F. S. P., Luis F. O. G., Claudia C. P. J., Walter M. A., John J. M. A., Phytochemical screening, free radical scavenging, and anti-inflammatory activity of Croton leptostachyus Kunth leaf extracts, Asian J. Pharm. Clin. Res, 2015, 8, 197-201.

[14] Elufioye T. O. Obuoror E. M., Sennuga A. T., Agbedahunsi J. M., Adesanya S. A.,, Acetylcholinesterase and butyrylcholinesterase inhibitory activity of som selected Nigerian medicinal plants, Brazilian journal of pharmacognosy 2010, 20 (4), 472-477.

[15] Bakthir H. Awadh Ali N. A., Arnold N., Teichert A. Wessjohann L., Anticholinesterase activity of endemic plant extracts from Soqotra, Afr. J. Tradit. Complement. Altern. Med. 2011, 8 (3): 296-299.

[16] Myung-Hee K., Sung-Hoon J., Hae-Dong J., Mee Sook L., Young-In K., Antioxidant activity and $\alpha$-glucosidase inhibitory potential of onion (Allium cepa L.) extracts. Food Sci Biotechnol., 2010, 19, 159-64.

[17] Owen, P. L.; Johns, T. Xanthine oxidase inhibitory activity of northeastern North American plant remedies used for gout. $J$. Ethnopharmacol. 1999, 64, 149-160.

[18] Umamaheswari, M., AsokKumar, K., Somasundaram, A., Sivashanmugam, T., Subhadradevi, V.; Ravi, T. K. Xanthine oxidase inhibitory activity of some Indian medical plants. $J$. Ethnopharmacol., 2007, 109, 547-551.

[19] Hoorn D. E. C. V. Nijiveldt R. J., Leeuwen P. A. M. V. Hofman Z., M'Rabet L., Bont D. B. A. D., Norren K. V., Accurate prediction of xanthine oxidase inhibition based on the structure of flavonoids, Eur. J. Pharmacol 2002, 451: 111118 . 